\title{
Comparing Coordination Abilities of 10-11 Years Old Girls Practicing Basketball and Rhythmic Gymnastic
}

\author{
Georgi Venelinov Brestnichki \\ Department of Basketball, Volleyball, Handball, National Sports Academy “Vasil Levski”, Sofia 1700, Bulgaria
}

\begin{abstract}
The words - coordination, coordination abilities and coordination training are getting more and more popular among the coaches in different sports. The rhythm makes the movement logical, easy and correct done. The purpose of this study is to determine any difference between the coordination abilities of 10-11 years old girls practicing basketball and rhythmic gymnastics. In that study, we have tested 40 girls at the age of 10-11 years practicing basketball and rhythmic gymnastic. We have tested them by 7 coordination tests. The results from the tests have been calculated in math-statistic methods and we have used descriptive statistic and $t$-test by Student with guaranteed probability $\mathrm{Pt} \geq 95 \%$. Ten-eleven old girls practicing rhythmic gymnastic have advantage against the girls practicing basketball in all 7 tests examining coordination abilities. The girls practicing rhythmic gymnastic, guaranteed by statistic, have better results in the balance and rhythmic abilities than the girls practicing basketball game. That statement is showing us that at this age the rhythmic gymnastic or elements from rhythmic gymnastic should be used for developing balance and rhythmic ability, which are very important for the basketball game.
\end{abstract}

Key words: Comparing, coordination, 10-11 years old girls, basketball, rhythmic gymnastics.

\section{Introduction}

The coordination abilities in sports are matter of discussion and researches in the last 10-15 years in among the coaches.

According to Smochevski [1], coordination of movement is important for regulation of motive action. The rhythm makes the movement logical, easy and correct done.

According to Bachvarov [2], coordination abilities include the ability to learn and perform difficult movements, as well as to be able to correct mistakes.

Basketball and rhythmic gymnastic are sports known with their coordination difficulty. In both sports coordination abilities have major role for the good performance.

Atanasov [3] recommends the usage of test battery to examine children's physical condition who is practicing rhythmic gymnastic. In this list of tests are included: balance on a right and left leg with closed

Corresponding author: Georgi Venelinov Brestnichki, Ph.D., research fields: sports and basketball. E-mail: gvbrestnichki@yahoo.com. eyes, put in order pulleys - examine the coordination and the speed for space motive reaction. The balance stability measured by a balance stand in position "antityuz" is better for the children practicing gymnastic than other sports and it is proofed by the statistic. These tests used by Atanasova shows that other coaches and specialists are interested in coordination abilities and they do different researches, in order to improve these abilities in their athletes.

G. Brestnichki [4] compares coordination abilities of 10-11 years old basketball and handball players, two sports which are very close in their structure and mechanic. He determines that the basketball athletes have better coordination abilities than handball once, but these results are not guaranteed by statistic. The results corroborate similarity of both sports.

The purpose of this study is to determine any difference between the coordination abilities of 10-11 years old girls practicing basketball and rhythmic gymnastic. Rhythmic gymnastic is a sport well known with high demands of coordination ability. And basketball is another sport, for which specialists 
recommended it for well development of the human body, his mentality and movement.

To achieve this goal, to determine which of these sports develop better coordination abilities at this age, we set up the following tasks.

1. To determine the coordination ability level of 10-11 years old girls practicing basketball and rhythmic gymnastics.

2. To compare the coordination abilities of 10-11 years old girls practicing basketball and rhythmic gymnastics.

\section{Methods}

In that study, we have tested 40 girls at the age of 10-11 years practicing basketball game and rhythmic gymnastic. Out of these 40 girls, 21 have practiced basketball and all the rest 19 have practiced rhythmic gymnastic. We have tested them by 7 coordination tests. The used test has been probated by Naumovski et al. [5]. The tests have guaranteed probability. They are separated in four groups-accuracy, balance, rhythmic abilities and coordination.

First group of tests is accuracy in movement with arms and legs: (1) Test 1: Aiming a target with a short bar using one hand (aiming a target with one hand); (2) Test 2: Aiming a target with tennis ball using the internal part of the foot (aiming a target with a leg).

Second group of tests is body balance: (1) Test 3: Balance on one leg alongside the equipment (balance stand) with opened eyes; (2) Test 4: Balance on one leg across the equipment (balance stand) with open eyes.

Third group of tests is rhythmic ability: (1) Test 5: Buffets with both hands without rhythm (rythmless buffet); (2) Test 6: Buffets with legs and arms on the wall (buffets with legs and arms).

Fourth group of test is coordination of the body: Test 7: Running in the figure of eight under a rope (running in figure eight).

The results from the tests have been calculated in math-statistic methods and we have used descriptive statistic and $t$-test by Student with guaranteed probability $\mathrm{Pt} \geq 95 \%$.

\section{Results}

The average results of the tested groups in accuracy and balance abilities are shown in Fig. 1 .

First group of tests examine the accuracy with arms and legs. The accuracy with the arms in the basketball game is well known. It is very important for the good shoot, for the accurate pass to a teammate. In the rhythmic gymnastic, the precision in the performance is extremely important-how the athlete will throw the equipment and how it would be caught after that. All these movement and the accuracy with the arms are important for the final result. The average results of the two tested groups in the accuracy abilities with the arms and legs, and the balance abilities are shown in Fig. 1. In the accuracy with the arms rhythmic gymnastic athletes have shown better results with 0.26 points than the basketball athletes. That difference has not been proofed by $t$-test of Student $\left(\mathrm{P}_{\mathrm{t}}=59.4 \%\right)$. Because of this statistical results, we could not proof that one of these two sports develop better accuracy with the arms.

In the accuracy with the legs, it is not that important for the basketball athletes as it is important for the rhythmic gymnastic ones, because they have situations
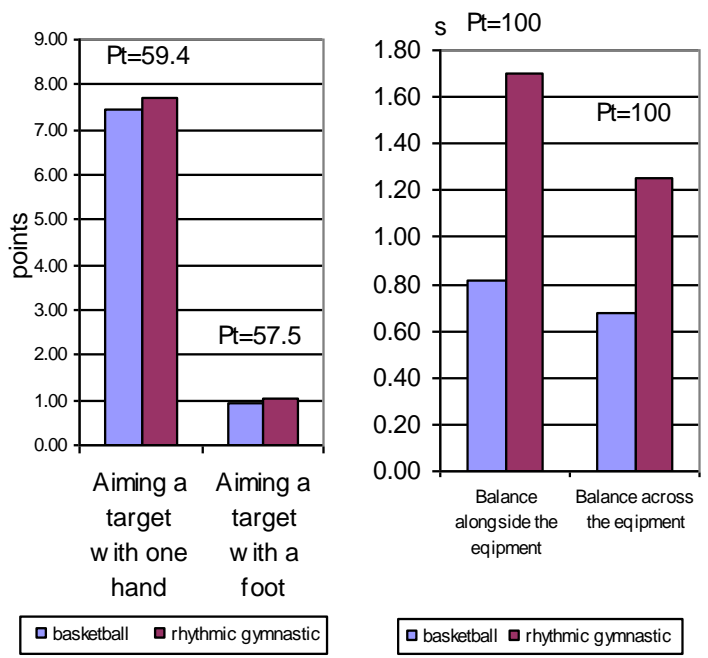

Fig. 1 Comparing aiming and balance abilities between athletes and pupils. 
in which they stop the equipment with the foot. According to the results, again the rhythmic gymnastic athletes have advantage in the shown abilities compare to the basketball athletes. The advantage in the results is very small -0.08 points and it is not guaranteed by the necessary probability. That statement does not allow us to tell which of the two sports develop better accuracy with arms and legs.

Next group of tests examine the balance abilities. Balance one of the major human abilities needed for the everyday live. In sports, it has an important role for better movements, for better drills and better performance. In the test measuring the balance abilities alongside the tested equipment, the girls practicing rhythmic gymnastic have shown better results compare to the girls practicing basketball. The difference in the results is $0.88 \mathrm{~s}$. This difference has the necessary statistic probability and we could say that practicing rhythmic gymnastic could develop better balance abilities, compared to practicing basketball game. This statement is also proofed by the second test measuring the balance abilities by balance on one leg across the equipment, in which the rhythmic gymnastic athletes have achieved again better result than the basketball athletes, with difference of $0.57 \mathrm{~s}$. That difference in the average results of both groups has been proved by statistic. The conclusion is that if we want the basketball athlete to improve their balance abilities (shooting, passing, defense stand and so on), we need to include specific exercises copied from the rhythmic gymnastic.

The results from tests examining the rhythmic abilities with the legs and arms and body coordination are shown in Fig. 2. The gymnastic sports are well known with its rhythmical characteristic. Also the rhythm or more likely the changes in rhythm are very important for the team sports in order to successful outplay the opponent. Comparing the rhythmic ability with arms of the girls practicing rhythmic gymnastic to these of girls practicing basketball, we found out that the difference in the average results is 1.74 cycles. According to the statistic calculations, the difference is proofed between both groups and it define the rhythmic gymnastic sport as better sport for developing rhythmic abilities with the arms.

About the second test examining the rhythmic abilities with arms and legs, 10-11 years old girls practicing rhythmic gymnastic has shown again better result than the girls same age practicing basketball game. The difference between the results is 1.91 cycles, which is significant. Also this difference has been proofed with high probability percent. From the shown results, we could conclude that practicing rhythmic gymnastic will develop better rhythmical abilities in the athletes.

The last test group measured coordination abilities of the body. This ability is very important for moving of the athletes on the field - for rational movements with better efficiency. In this test, the girls practicing rhythmic gymnastic have shown better results than the girls practicing basketball game. This result shows us that high variation of movement present in the rhythmic gymnastic helps better development of body coordination in the participants. But at the same time, in basketball game, situations are change continuously and the athletes need to take an action immediately
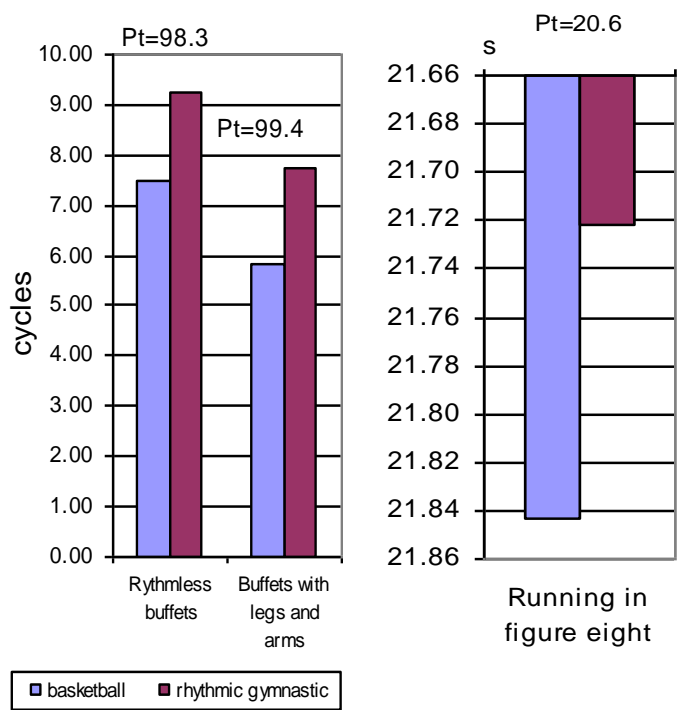

Fig. 2 Comparing rhythm and coordination abilities of athletes and pupil. 


\section{Practicing Basketball and Rhythmic Gymnastic}

according to the situation in order to beat the opponent.

Solution in this controversy between these two sports is found. The difference between the average results of the groups is $0.12 \mathrm{~s}$. That difference is very low and according to the low guaranteed percentage- $20.6 \%$, we could not define which of the tested sports could gain better coordination ability of the body. Or maybe both sports develop good body coordination in movement.

\section{Conclusion}

Ten-eleven old girls practicing rhythmic gymnastic have advantage against the girls practicing basketball in all 7 tests examining coordination abilities.

The girls practicing rhythmic gymnastic, guaranteed by statistic, have better results in the balance and rhythmic abilities than the girls practicing basketball game. That statement is showing us that the rhythmic gymnastic or elements from rhythmic gymnastic should be used for developing balance and rhythmic ability at this age.

\section{References}

[1] Smochevski, M. 2001. "Development of Coordination Abilities and Motive Habits in Pupils and Children Practicing Basketball at the Age of 10-11 Years." Ph.D. thesis, The National Sports Academy, Sofia, Bulgaria.

[2] Bachvarov, M. 2000. Sport-logic. Sofia: NSA-press. 3. Atanasov, T. 1984. Subject Planning of the Early Preparation for Rhythmic Gymnastics. Coaching Thoughts, Vol. 4. Sofia: National Sports.

[3] Brestnichki, G. 2005. "Comparing of Coordination Abilities of 10-11 Years Old Girls Practicing Basketball and Handball." Sport and Sience 4: 27-30.

[4] Naumovski, Al., Matovski, St., Georgiev, G., Tsvetkov, Vl., Mineva, M. 2004. Measure Characteristics of some Test Evaluating Motor Latent Dimension. Skopje. PAKUNG. 